\title{
A Convex Relaxation Approach to Fat/Water Separation with Minimum Label Description
}

\author{
Abraam S. Soliman, Jing Yuan, James A. White, \\ Terry M. Peters, and Charles A. McKenzie \\ University of Western Ontario, London, ON, Canada \\ Robarts Research Institute, London, ON, Canada
}

\begin{abstract}
While Magnetic Resonance Imaging is capable of separating water and fat components in the body, mapping of magnetic field inhomogeneities is essential for the successful application of this process. In this study, we address the problem of field map estimation using a convex-relaxed max-flow method. We propose a novel two-stage approach that leads to the global optimum of the proposed problem. The first stage minimizes the signal residuals via a convexrelaxed minimum description length (MDL)-based approach. The MDL-based labeling model penalizes the total number of appearing labels, which helps to avoid field map errors when abrupt changes in field homogeneity exist. By exploring the whole range of possible frequency offsets, this stage ensures limiting the estimated field offset within certain boundaries where the global minimum resides. The second stage employs the output of the labeling model in a commonly used gradient-descent based method (known as IDEAL) to converge to the exact global minimum, i.e. the required value of the field offset. Experimental results for cardiac imaging, where challenging field inhomogeneities exist, showed that our method significantly outperforms over a widely-used technique for fat/water separation in terms of robustness and efficiency.
\end{abstract}

\section{$1 \quad$ Introduction}

The ability to separate fat from water in a magnetic resonance (MR) image is an important problem for a number of clinical applications. Bright fat signal can obscure underlying pathology and therefore suppression of the fat signal is required. In other cases, fat is considered an important diagnostic marker, and hence a clearer depiction of its signal, rather than its suppression, is desired. Common clinical applications of the latter include the diagnosis of non-alcoholic fatty liver diseases (NAFLD) [1], as well as a variety of bone marrow diseases [2]. However, expanding interest in the evaluation of myocardial fat infiltration and pericardial fat volume [3] justifies its optimization for cardiac imaging.

Among various available MR techniques, chemical-shift based (or Dixon-based) techniques have become the most commonly used methods to obtain a quantitative fat measurement [4]. Chemical-shift based methods are characterized by their unique ability to extract a fat-only image with positive contrast, unlike other techniques that either tend to suppress the fat signal, making the process of identifying fat voxels 
ambiguous, or apply fat selective excitation which is sensitive to B0 and B1 inhomogeneities.

Unfortunately, a successful fat/water separation with Dixon-based techniques relies largely on the homogeneity of the magnetic field. In other words, the mapping of the magnetic field inhomogeneities - so called field map, cannot be decoupled from the fat/water separation process ( $\operatorname{see} \operatorname{Sec} 2.1$ ). The field map estimation problem therefore, leads to a non-linear non-convex optimization problem, which has multiple local minima. An error in estimating the field map would propagate to the resultant water and fat images, causing what we term fat/water swaps. A "swap" is defined as assigning the main signal in a water-dominant voxel as fat, or vice-versa - an example of fat/water swaps is shown in Fig.1.
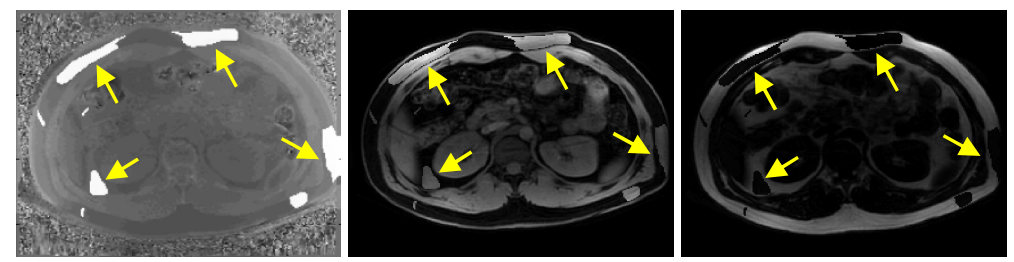

Fig. 1. Left to right: Fat/water swaps appearing in field map, water and fat components

A commonly-used technique in fat/water separation is the "Iterative Decomposition of water and fat with Echo Asymmetry and Least square estimation", abbreviated as IDEAL [5]. IDEAL is a Dixon-based method that acquires at least three echoes to estimate the field map, water and fat components. However, IDEAL is a local optimization method that heavily depends on the initialization process, and hence, convergence to the global optimum is not guaranteed. Moreover, it is a voxel-independent optimization, i.e. it does not enforce any global smoothness prior to the estimated field map. Yu et al. [6] proposed a region-growing technique ${ }^{1}$ to address the flaws of IDEAL. This method implicitly imposes a spatial smoothness on the field map; however, it does not account for the abrupt changes in magnetic field that might exist at tissue/air interfaces, which might cause fat/water swaps. Although several techniques have been proposed in the literature [6-10] to address the field map estimation problem, only a few have been considered sufficiently robust for clinical use [8, 11]. Further, their application has been limited to 1.5 Tesla [11] where B0 field inhomogeneities are modest compared to higher field strengths.

In this work, we propose a novel field map estimation approach that can withstand abrupt changes in field homogeneity at higher field strengths, particularly at 3.0 Tesla, while guaranteeing smoothness of the estimated field map. Our method relies on prior knowledge of the periodic variation of signal residuals with the field map values [68]. We use a two-stage approach to reach the global minimum solution, and provide a high resolution mapping of the field inhomogeneities. First, a label-cost prior maxflow approach [12] is performed on the signal residues to converge near the global

\footnotetext{
${ }^{1}$ This nomenclature should not be confused with the conventional "region-growing" method used in general image processing applications.
} 
optimum. The output is employed as an initial guess to the second stage, where a conventional gradient-descent IDEAL is applied to reach the exact field offset. Our method is tested for cardiac as well as abdominal images obtained at 3.0 Tesla, where challenging B0 field inhomogeneities commonly exist. Comparing to the region growing method [6], our approach has significantly improved the robustness of field map estimation process and has efficiently removed fat/water swaps.

\section{Theory and Methodology}

In the following sections, we first derive the signal equation to be minimized; then we introduce the multi-labeling convex relaxation model and its dual continuous maxflow formulation along with the minimum description length (MDL) principle, which are applied in the first stage of our approach. The proposed MDL-based labeling model penalizes the number of "appearing" labels, which helps to avoid the small regional fat/water swaps that might appear in the presence of severe and rapid changes of magnetic field. In other words, such MDL prior smooth out small-scale partitions, which usually correspond to regional fat/water swaps. The MDL-based labeling model results in a coarse estimation of the field map. This step guarantees a global minimization by labeling each pixel with a field map value located near its global optimum solution. The coarse estimate of field map serves as an initial guess for the second stage that consists of applying the IDEAL iterative process $[5,13]$. A stopping criterion of $<1 \mathrm{~Hz}$ was used for the iterative process, in order to provide a field map with a sufficient resolution for clinical applications, particularly pericardial fat quantification. Once the final field map is obtained, water and fat components can be directly computed from Eq.2.

\subsection{Signal Equation}

Let $S($.$) denote the signal acquired from a voxel v$, containing a mixture of water and fat, such that:

$$
S_{v}\left(t_{n}\right)=\left(\rho_{W, v}+\rho_{F, v} \cdot \sum_{m=1}^{M} \alpha_{m} \cdot e^{i 2 \pi \delta_{m} t_{n}}\right) \cdot e^{i 2 \pi \varphi_{v} t_{n}}
$$

where $t_{n}$ denotes the echo-time (TE) shift $(n=1, \ldots, N)$ of the acquired signal; $\rho_{W, v}$ and $\rho_{F, v}$ are the water and fat components at voxel $v$, respectively; $M$ is the number of fat peaks in the fat spectrum; $\delta_{m}$ is the frequency of the $m$-th peak with its corresponding amplitude $\alpha_{m}(\mathrm{~Hz})$, such that $\sum_{m=1}^{M} \alpha_{m}=1 ; \varphi_{v}(\mathrm{~Hz})$ is the local frequency offset at voxel $v$ (i.e. the field map). We used a calibrated fat spectrum model as shown in [14], where $M=6$ and the main fat peak is at $\sim 420 \mathrm{~Hz}$, relative to the water peak at 3.0 Tesla. Having three or more echo-times (TE) acquired (as described above), Eq.1 can be reformulated as follows:

$$
\mathrm{S}_{v}(t)=\Psi\left(\varphi_{v}, t\right) \cdot \mathrm{A}(t) \cdot \mathrm{P}_{v},
$$


where

$$
\Psi\left(\varphi_{v}\right)=\left[\begin{array}{ccc}
e^{i 2 \pi \varphi_{v} t_{1}} & 0 & 0 \\
0 & \ddots & 0 \\
0 & 0 & e^{i 2 \pi \varphi_{v} t_{N}}
\end{array}\right], \mathrm{A}=\left[\begin{array}{cc}
1 & \sum_{m=1}^{M} \alpha_{m} \cdot e^{i 2 \pi \delta_{m} t_{1}} \\
\vdots & \vdots \\
1 & \sum_{m=1}^{M} \alpha_{m} \cdot e^{i 2 \pi \delta_{m} t_{N}}
\end{array}\right],
$$

$\mathrm{S}_{v}(t)=\left[S_{v}\left(t_{1}\right), \ldots S_{v}\left(t_{n}\right)\right]^{T}, \mathrm{P}_{v}=\left[\rho_{W, v}, \rho_{F, v}\right]^{T}$. To estimate the required water and fat components $\left(\rho_{W, v}, \rho_{F, v}\right)$, the frequency offset $\varphi_{v}$ should be demodulated first. Hence, dropping the known echo-time shift $\left(t_{n}\right)$, a non-linear least-squares cost function can be derived from (2) as follows:

$$
\begin{gathered}
\Gamma\left(\varphi_{v}\right):=\left\|\mathrm{A} \cdot \widehat{\mathrm{P}_{v}}-\widehat{\Psi}^{-1}\left(\varphi_{v}\right) \cdot \mathrm{S}_{v}\right\|_{2} \\
\Gamma\left(\varphi_{v}\right):=\left\|\left(\mathrm{AA}^{\dagger}-I\right) \widehat{\Psi}^{-1}\left(\varphi_{v}\right) \cdot \mathrm{S}_{v}\right\|_{2},
\end{gathered}
$$

where $\widehat{\mathrm{P}_{v}}$ and $\widehat{\Psi}\left(\varphi_{v}\right)$ are the estimated values of $\mathrm{P}_{v}$ and $\Psi\left(\varphi_{v}\right)$ respectively, $I$ is the identity matrix, and ${ }^{\dagger}$ denotes the pseudo-inverse, s.t. $\mathrm{A}^{\dagger}=\left[\mathrm{A}^{T} \mathrm{~A}\right]^{-1} \mathrm{~A}^{T}$. However, two main problems are encountered when minimizing $\Gamma\left(\varphi_{v}\right)$ : first, the non-convex property of the function, and second, it does not impose a priori smoothness on the estimated field map, as it is a voxel-by-voxel based strategy, and global minimization is not guaranteed. We address these problems by using a convex-relaxation approach that guarantees the global minimization, and implicitly includes the required smoothness of the field map.

It is important to note that, to maximize the signal-to-noise performance, the images are acquired at equally-spaced echo-time shifts (i.e. $\mathrm{TE}_{\mathrm{n}}-\mathrm{TE}_{\mathrm{n}-1}=\Delta \mathrm{TE}$ ) [7]. In this case, $\Gamma\left(\varphi_{v}\right)$ is periodic with a period of $1 / \Delta \mathrm{TE}[6,7]$. This allows us to determine the lower and upper bounds, which are set to $[ \pm 1 /(2 \Delta \mathrm{TE})]$, necessary for the max-flow model used in the first stage. The whole range is divided into $\sim 20$ equallyspaced values, which are used to label the input cost function, as described below.

\subsection{A Continuous Max-Flow Approach to MDL-Based Potts Model}

The Potts Model: In image processing, a multi-labeling problem assigns the optimal label $\varphi \in \varphi_{1} \ldots \varphi_{L}$ to each voxel. The Potts model is a labeling approach that minimizes the total perimeter of all one-label regions, without assuming any prior order for the labels. It results in a partition of the continuous domain $\Omega$ into $L$ disjoint subdomains $\left\{\Omega_{i}\right\}_{i=1}^{L}$, as follows:

$$
\begin{gathered}
\min _{\left\{\Omega_{i}\right\}_{i=1}^{L}} \sum_{i=1}^{L} \int_{\Omega_{i}} \Gamma\left(\varphi_{i}, x\right) d x+\lambda \sum_{i=1}^{L}\left|\partial \Omega_{i}\right| \\
\text { s.t. } \bigcup_{i=1}^{L} \Omega_{i}=\Omega \text {, and } \Omega_{k} \bigcap \Omega_{m}=\phi, \quad \forall k \neq m
\end{gathered}
$$


where $\Gamma\left(\varphi_{i}, x\right)$ is the cost of assigning label $\varphi_{i}$ to location $x$, as defined in Eq.3, and $\left|\partial \Omega_{i}\right|$ measures the perimeter of each subdomain $\Omega_{i}, i=1, \ldots, L$.

The Potts model in Eq.4 can be efficiently solved by its convex-relaxation model as follows [15]:

$$
\min _{u \in S}\left\{\mathrm{E}(u):=\sum_{i=1}^{L}\left(\int_{\Omega} u_{i}(x) \Gamma\left(\varphi_{i}, x\right) d x+\beta \int_{\Omega}\left|\nabla u_{i}\right| d x\right)\right\}
$$

where $S$ is the convex constrained set of $u(x):=\left(u_{1}(x), \ldots, u_{L}(x)\right)$ :

$$
S=\left\{u(x) \mid \sum_{i=1}^{L} u_{i}(x)=1, \quad \forall x \in \Omega ; \quad u_{i}(x) \in[0,1], \quad i=1, \ldots, L\right\}
$$

Minimum Description Length (MDL)-Based Potts Model: The minimum description length (MDL) principle penalizes the number of appearances or labels in image labeling problems. It naturally leads to the use of fewer partitions or labels to describe the given image, without simultaneously over smoothing the underlying domain [12]. The MDL cost is introduced by adding the term $\gamma Z$ to the Potts model, where $Z=\#\left\{1 \leq i \leq L \mid \Omega_{i} \neq \emptyset\right\}$ gives the number of non-empty labels, i.e. a label-cost prior. Yuan et al. [12] showed that adding the label-cost prior to Eq.5 leads to the following convex-relaxed MDL-based Potts model, used here:

$$
\min _{u \in S}\left\{\mathrm{E}(u):=\sum_{i=1}^{L}\left(\int_{\Omega} u_{i}(x) \Gamma\left(\varphi_{i}, x\right) d x+\beta \int_{\Omega}\left|\nabla u_{i}\right| d x\right)\right\}+\gamma \sum_{i=1}^{L} \max _{x \in \Omega} u_{i}(x)
$$

Fast Continuous Max-Flow Approach: The continuous max-flow approach [15, 16] to the MDL-based Potts model (Eq.6) used in this study is summarized below:

Let $\Omega$ be a continuous $2 \mathrm{D}$ image domain, $L$ the number of labels, and $\Omega_{i}, i=$ $1, \ldots L$ a copy of $\Omega$ assigned to the $i^{\text {th }}$ label. For each location $x \in \Omega$, a source flow $\rho_{s}(x)$ is streaming from the source node $s$ to a labeled copy $\Omega_{i}$, s.t. $\forall \Omega_{i}, i=$ $1, \ldots L, \rho_{s}(x)$ is the same. Similarly for each $x \in \Omega$, a sink flow to the sink $t$ is assigned. However, $\rho_{i}(x), i=1, \ldots L$ may differ. A spatial flow $q_{i}(x) i=1, \ldots L$ is also defined for each location. The continuous max-flow model can be formulated as follows:

$$
\max _{\rho_{s}, \rho, q}\left\{P\left(\rho_{s}, \rho, q\right):=\int_{\Omega} \rho_{s} d x\right\},
$$

subject to the constraints:

a. $\left(\operatorname{div} q_{i}-\rho_{s}+\rho_{i}-r_{i}\right)(x)=0, \quad i=1, \ldots, L$

b. $\left|q_{i}(x)\right| \leq C_{i}(x), \quad \rho_{i}(x) \leq \Gamma\left(\varphi_{i}, x\right), \int_{\Omega}\left|r_{i}(x)\right| d x \leq \gamma \quad i=1, \ldots, L$, 
where $C_{i}(x)=\beta$ is the capacity of the spatial flow $q_{i}(x)$, and $r_{i}(x)$ is the extra flow associated with the penalty of the number of labels. Yuan et al. [12] proved that the max-flow formulation (Eq.7) is dual to the convex-relaxed MDL-based Potts model, and results in an efficient flow maximization algorithm to Eq.6.

\section{Experiments and Discussion}

Our method was tested on 19 abdominal and cardiac images acquired on a 3.0 T MRI system (Discovery MR 750, GE Healthcare, Waukesha, WI). Cardiac images were acquired in different orientations (short-axis, 4-chambers and axial views) with a fast multi-echo GRE sequence using a 32-coil cardiac array [17]. Abdominal images were acquired with a 3D IDEAL-SPGR sequence [14] using an 8-coil array. Four and 6 equally-spaced echo-time shifts were used (interleaved acquisition for cardiac data). Each cardiac slice is acquired in one 20 s breath-hold while the 8 abdominal slices were acquired with parallel MRI acquisition (acceleration factor of 2) in one $20 \mathrm{~s}$ breath-hold. Matrix sizes of acquired images varied between 256x256 and 256x192.

The field map estimated from the first and final stages of our approach, as well as the fat and water components are shown below (Fig.2). For the abdominal example, $\mathrm{TE} / \Delta \mathrm{TE}=1.04 / 0.828 \mathrm{msec}$ and $\mathrm{TR}=7.324 \mathrm{msec}$; for the cardiac example, $\mathrm{TE} / \Delta \mathrm{TE}=$ 2.28/1.54 msec and TR $=12.66 \mathrm{msec}$.
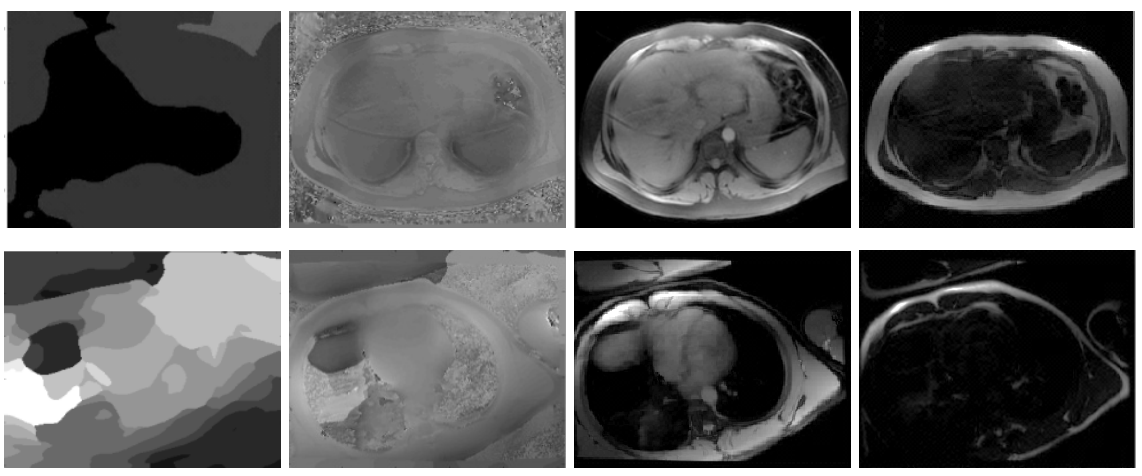

Fig. 2. Upper row: axial abdominal slice, lower row: 4-chambers cardiac view. Left to right: the coarse estimate of field map from the max-flow stage, final field map after the second stage, water and fat components.

Our results are compared to those obtained from the region-growing technique [6] on the same dataset, to show that fat/water swaps have been significantly reduced (Fig.3). A major drawback in the region-growing method is that it forces the field map smoothness using a 2D extrapolation approach, which does not account for the abrupt changes of field homogeneity, in particular in cardiac images. We have tackled this problem by employing a convex-relaxed labeling model that guarantees the global optimum. Moreover, it implicitly imposes smoothness on the estimated field map by penalizing small partitions, which correspond to regional fat/water swaps. 

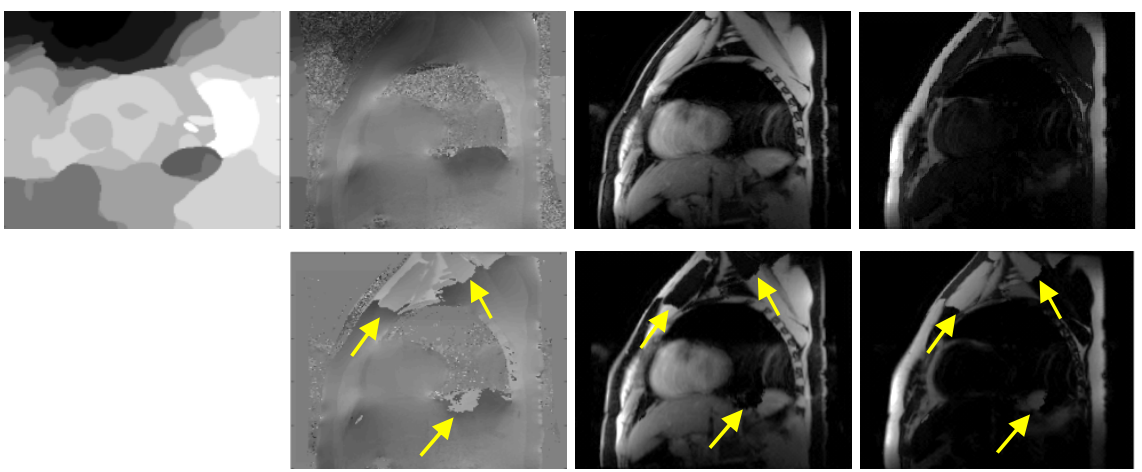

Fig. 3. Comparison between our approach (upper row) and the region-growing method (lower row) on a short-axis cardiac image. Yellow arrows indicate the locations of fat/water swaps that have clearly avoided by our method.

The processing time of our method is 1.5-5 minutes per image, depending on the level of smoothness and the underlying degree of inhomogeneities, compared to 6.5 minutes per image for the region-growing method. Moreover, the max-flow stage can be easily accelerated over modern computation frameworks, e.g. graphics processing units (GPU).

Taking into account the periodicity of $\Gamma(\varphi)[6,7]$ (i.e. $[ \pm 1 /(2 \Delta \mathrm{TE})]$ ), we found that using 20 equally-spaced field offsets for the first stage is sufficient to target the optimal label. However, relying only on the max-flow stage to reach the exact field offset would significantly increase the processing time, as we would use up to $\sim 800$ labels in order to achieve the same high resolution field map.

The robustness of field map estimation techniques is usually judged by the visual identification of fat/water swaps. However, we provide an approximate metric for the effectiveness of the technique by counting the number of pixels that have shown fat/water swaps in a user-defined region of interest - In abdominal images, the liver is assumed to be the region of interest, while in cardiac images the whole intra-thoracic space including the area surrounding the diaphragm is considered. An approximate average for the pixels showing fat/water swaps is $\sim 274 \pm 720$ pixels with the regiongrowing technique vs. $\sim 17 \pm 32$ pixels with our proposed method.

Comparing to a recent graph cut-based technique [8] currently used in clinical applications [11], our approach provides a field map with higher frequency resolution, with an estimation error less than $1 \mathrm{~Hz}$ over the whole image. This may have important utility for supporting accurate myocardial and pericardial fat quantification, given an expanding use of 3.0 Tesla field strengths for cardiac imaging.

Acknowledgments. We acknowledge funding from CIHR Strategic Training Program for Vascular Research, and Ontario Research Fund: Imaging for Cardiovascular therapeutics. This research was undertaken, in part, thanks to funding from the Canada Research Chairs program. We thank Karl K. Vigen for providing cardiac IDEAL pulse sequence, Curtis Wiens for helping in data acquisition, and Bryan Addeman for the revision of the manuscript. We acknowledge the use of the Fat-Water Toolbox (http://ismrm.org/workshops/FatWater12/data.htm) for some of the experiments. 


\section{References}

1. Lall, C.G., Aisen, A.M., Bansal, N., Sandrasegaran, K.: Nonalcoholic fatty liver disease. Am J. Roentgeno. 190, 993-1002 (2008)

2. Haavardsholm, E.A., Bøyesen, P., Østergaard, M., Schildvold, A., Kvien, T.K.: Magnetic resonance imaging findings in 84 patients with early rheumatoid arthritis: bone marrow oedema predicts erosive progression. Ann. Rheum. Dis. 67, 794-800 (2008)

3. Iozzo, P.: Myocardial, Perivascular, and Epicardial Fat. Diabetes Care 34, S371 (2011)

4. Ma, J.: Dixon techniques for water and fat imaging. Magn. Reson. Imaging 28, 543-558 (2008)

5. Reeder, S., Pineda, A., Wen, Z., Shimakawa, A., Yu, H., Brittain, J., Gold, G., Beaulieu, C., Pelc, N.: Iterative decomposition of water and fat with echo asymmetry and leastsquares estimation (IDEAL): Application with fast spin-echo imaging. Magn. Reson. Med. 54, 636-644 (2005)

6. Yu, H., Reeder, S., Shimakawa, A., Brittain, J., Pelc, N.: Field map estimation with a region growing scheme for iterative 3-point water-fat decomposition. Magn. Reson. Med. 54, 1032-1039 (2005)

7. Lu, W., Hargreaves, B.: Multiresolution field map estimation using golden section search for water-fat separation. Magn. Reson. Med. 60, 236-244 (2008)

8. Hernando, D., Kellman, P., Haldar, J., Liang, Z.: Robust water/fat separation in the presence of large field inhomogeneities using a graph cut algorithm. Magn. Reson. Med. 63, 79-90 (2010)

9. Soliman, A.S., Yuan, J., Vigen, K., White, J.A., Peters, T.M., McKenzie, C.A.: Robust Field Map Estimation using VARPRO and Multi-labeling Continuous Max-Flow. In: Proc. 20th ISMRM, p. 2508 (2012)

10. Soliman, A.S., Yuan, J., Vigen, K., White, J.A., Peters, T.M., McKenzie, C.A.: Fast Field Map Estimation withMulti-labeling Continuous Max-Flow. In: ISMRM Workshop on FatWater Separation (2012)

11. Kellman, P., Hernando, D., Arai, A.: Myocardial Fat Imaging. Current Cardiovascular Imaging Reports 3, 83-91 (2010)

12. Yuan, J., Bae, E., Boykov, Y., Tai, X.-C.: A Continuous Max-Flow Approach to Minimal Partitions with Label Cost Prior. In: Bruckstein, A.M., ter Haar Romeny, B.M., Bronstein, A.M., Bronstein, M.M. (eds.) SSVM 2011. LNCS, vol. 6667, pp. 279-290. Springer, Heidelberg (2012)

13. Reeder, S., Wen, Z., Yu, H., Pineda, A., Gold, G., Markl, M., Pelc, N.: Multicoil Dixon chemical species separation with an iterative least-squares estimation method. Magn. Reson. Med. 51, 35-45 (2004)

14. Yu, H., Shimakawa, A., McKenzie, C.A., Brodsky, E., Brittain, J.H., Reeder, S.B.: Multiecho water-fat separation and simultaneous R2* estimation with multifrequency fat spectrum modeling. Magn. Reson. Med. 60(5), 1122-1134 (2008)

15. Yuan, J., Bae, E., Tai, X.-C., Boykov, Y.: A Continuous Max-Flow Approach to Potts Model. In: Daniilidis, K., Maragos, P., Paragios, N. (eds.) ECCV 2010, Part VI. LNCS, vol. 6316, pp. 379-392. Springer, Heidelberg (2010)

16. Yuan, J., Bae, E., Tai, X.: A Study on Continuous Max-Flow and Min-Cut Approaches. In: Computer Vision and Pattern Recognition (CVPR), pp. 2217-2224 (2010)

17. Vigen, K.K., François, C.J., Yu, H., Shimakawa, A., Brittain, J.H., Reeder, S.B.: Multiecho IDEAL Cardiac Water-Fat Imaging. In: Proc. 17th ISMRM, p. 2775 (2009) 